\title{
Collagenous colitis with rapid response to sulphasalazine
}

\author{
T. Rokkas, ${ }^{1}$ M.I. Filipe ${ }^{2}$ and G.E. Sladen ${ }^{1}$ \\ ${ }^{1}$ Gastroenterology Unit, Division of Medicine and ${ }^{2}$ Department of Histopathology, UMDS Guy's and St \\ Thomas' Hospitals, Guy's Hospital, London SE1 9RT, UK.
}

\begin{abstract}
Summary: A case of collagenous colitis in a young female with a rapid response to sulphasalazine both symptomatically and histologically is reported. This is only the third such response to be reported. In most published accounts, collagenous colitis fails to respond to treatment and runs a very prolonged course.
\end{abstract}

\section{Introduction}

In 1976 Lindstrøm $^{1}$ reported the first case of a previously unrecognized entity which was characterized by the association of chronic, watery diarrhoea. and a marked deposition of collagen below the surface epithelium. He introduced the term 'collagenous colitis' for this unusual disease and since then approximately 30 cases have been described, mainly from Europe. ${ }^{2-12}$ The aetiology is unknown and no specific treatment is so far available. $^{8}$ Most reported cases have been given sulphasalazine but have failed to respond ${ }^{9}$ unlike 'microscopic colitis' which usually does respond.

We present a case of collagenous colitis with an impressive rapid symptomatic response to sulphasalazine and complete histological resolution of the collagen deposits which continued throughout the year's follow up.

\section{Case report}

A 33 year old white female patient was seen in November 1985 with a one year history of profuse watery diarrhoea (up to 20 stools/day) and intermittent colicky abdominal pain. She described the stools as frothy, without blood or mucus and not usually offensive. Otherwise her general health had been good until 1 month before her attendance when she started to lose weight ( $7 \mathrm{~kg}$ over 1 month) and felt tired. Anti-diarrhoeal drugs were ineffective. Physical examination was unremarkable except that the abdomen was a little distended and

Correspondence: G.E. Sladen D.M., F.R.C.P. Accepted: 18 June 1987 noisy. Sigmoidoscopy revealed markedly watery stools with rather oedematous but otherwise unremarkable mucosa. Rectal biopsy showed debasement of the surface epithelium with complete loss of goblet cells and infiltration by neutrophils. There was a thick irregular layer of collagen varying from 7 to $25 \mu \mathrm{m}$ that had replaced the normal basement membrane (Figure 1). It had a very coarsely fibrillar structure with its interstices lightly infiltrated by eosinophils, neutrophils lymphocytes and macrophages in small numbers No amyloid was demonstrated in a Congo red? preparation. Faecal cultures on several occasions were negative for pathogens during the few months before diagnosis. Haemoglobin, blood film, erythrocyte sedimentation rate and blood chemistry were normal. Barium enema and barium meal with follow through were also normal. The patient was treated with oral sulphasalazine $3 \mathrm{~g}$ /day and within a few days her diarrhoea and abdominal pain began to improve. By the end of one week she was completely free of symptoms and her bowel habit was normal (1-3 formed stools daily). Sulphasalazine was discontinued one month after the start of treatment without relapse. Six months after the initial presentation a rectal biopsy showed complete disappearance of the collagen layer and the mucosa appeared normal (Figure 2). One year after her initial presentation the patient remained free of symptoms and multiple rectal and colonic biopsies obtained by flexible sigmoidoscopy showed no evidence of the collagen layer or inflammation.

\section{Discussion}

The case we described has all the characteristics of collagenous colitis. The patient was female and it is

(C) The Fellowship of Postgraduate Medicine, 1988 


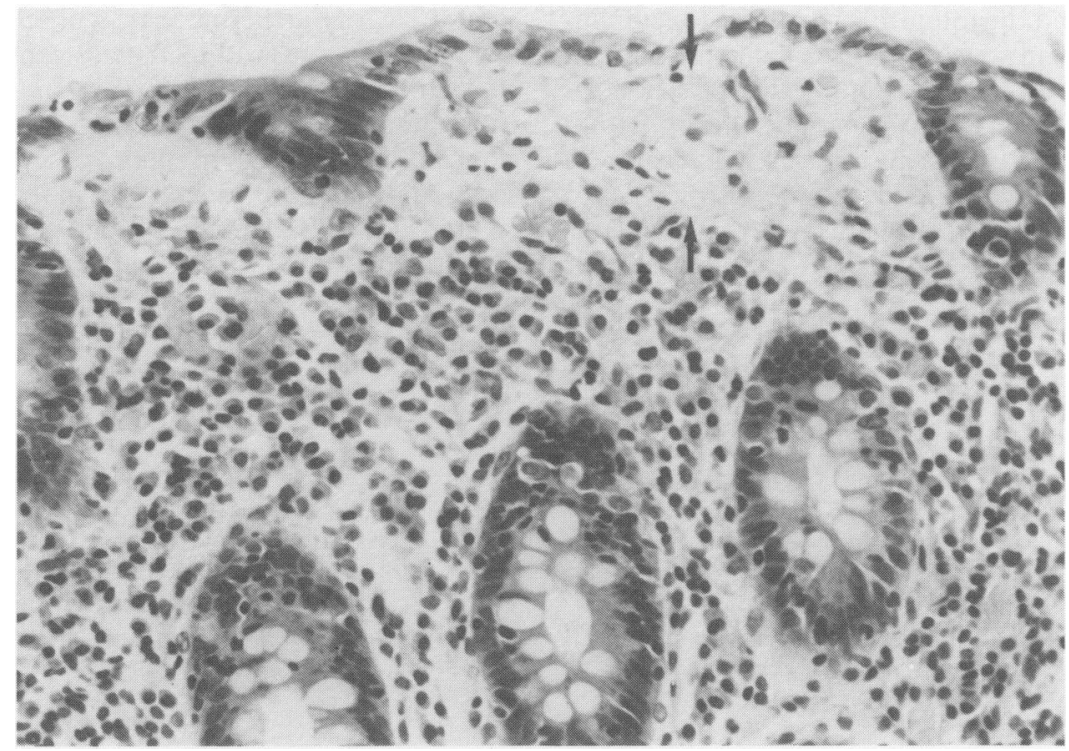

Figure 1 Microscopic appearance of the first rectal biopsy specimen which shows a dense band of collagen underlying all surface epithelium (between arrows). The lamina propria shows a moderate cellular infiltrate mainly of plasma cells. Haematoxylin-eosin stain; $\times 350$.

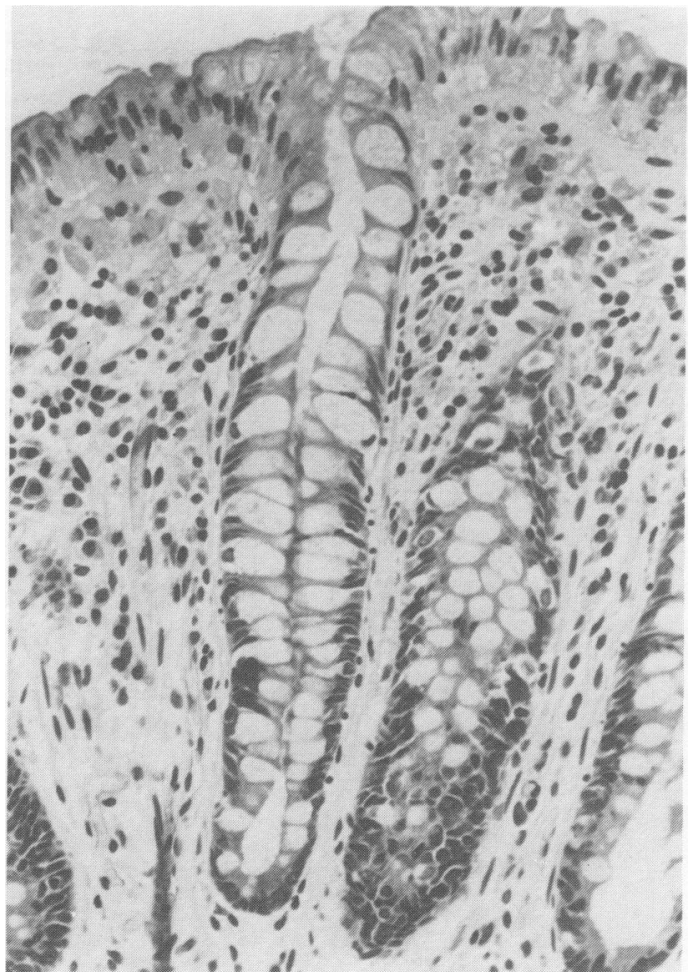

Figure 2 Second rectal biopsy following treatment shows return to normal histology. Haematoxylin-eosin stain; $\times 350$. known that the disease is much more common in women, ${ }^{9}$ although the reason for this is unknown. ${ }^{10}$ She presented with profuse watery diarrhoea and intermittent colicky abdominal pain which are the main clinical characteristics of the disease. ${ }^{9}$ Her first rectal biopsy showed a subepithelial collagen layer of 7 to $25 \mu \mathrm{m}$ in thickness which was well above the range of $0.4-4.6 \mu \mathrm{m}$ observed by Van den Oord et al. ${ }^{3}$ in 564 rectal biopsies from normal subjects (200), patients with inflammatory bowel disease (104) and patients with miscellaneous colonic disorders (260).

The aetiology of collagenous colitis is unknown ${ }^{9}$ but the most widespread hypothesis is that collagen accumulation results from increased synthesis and/or decreased cell turnover. ${ }^{11}$ When the cell turnover is decreased, fibrocytes remain longer in the mature phase and so produce more collagen. It is known that in the immature replicative phase fibroblasts produce very little collagen and in the colon it is only after they have moved to the crypt together with the epithelial cells that they are able to synthesize collagen. ${ }^{12}$ The possibility that an infectious factor is responsible has also been postulated by others ${ }^{4}$ who reported remission of one patient's symptoms and a reduction of collagen layer thickness after a course of mepacrine, a drug which is generally used as an antimicrobial agent.

So far there has been a widespread belief that there was no effective treatment for this disease. ${ }^{9}$ However, there are two reported cases with 
symptomatic and histological response to sulphasalazine $^{6,8}$ and in one ${ }^{8}$ sulphasalazine was given with local steroid therapy. Our patient responded impressively to sulphasalazine both clinically within the first week of treatment and histologically as proved by normal rectal and colonic histology at 6 and 12 months. The mechanism by which this treatment leads to dissolution of the collagen layer

\section{References}

1. Lindstrøm, C.G. Collagenous colitis with watery diarrhoea - a new entity? Pathol Eur 1976, 11: 87-89.

2. Bogomoletz, W.V., Adnet, J.J., Birembaut, P., Feydy, P. \& Dupont, P. Collagenous colitis: an unrecognised entity. Gut 1980, 21: 164-168.

3. Van den Oord, J.J., Geboers, K. \& Desmet, V.J. Collagenous colitis: an abdominal collagen table? Two new cases and review of the literature. $A m J$ Gastroenterol 1982, 77: 377-381.

4. Pieterse, A.S., Hecker R. \& Rowland, R. Collagenous colitis: a distinctive and potentially reversible disorder. J Clin Pathol 1982, 35: 338-340.

5. Rask-Madsen, J., Grove, O., Hausen, M.G.J., Buckhare, K. \& Henrik-Nielson, R. Colonic transport of water and electrolytes in a patient with diarrhoea due to collagenous colitis. Dig Dis Sci 1983, 28: 1141-1146.

6. Weidner, N., Smith, J. \& Pattee, B. Sulphasalazine in treatment of collagenous colitis: case report and review of the literature. Am J Med 1984, 77: 162-166.

7. Taglbjaerg, P.S. \& Thaysen, E.H. Collagenous colitis: an ultrastructural study of a case. Gastroenterology 1983, 82: 561-563. is not clear. Furthermore it is obscure which of the two constituents of sulphasalazine, i.e. sulphapyridine (antimicrobial agent) or salicylate (antiinflammatory agent), is responsible for this histological regression. In any case this regression suggests that the disease is not inevitably progressive and that sulphasalazine should prove to be effective treatment at least in some patients.

8. Farah, D.A., Mills, P.R., Lee, F.D., McLay, A. \& Russell, R.I. Collagenous colitis: possible response to sulphasalazine and local steroid therapy. Gastroenterology 1985, 88: 792-797.

9. Fausa, O., Foerster, A. \& Horig, T. Collagenous colitis. A clinical, histological and ultrastructural study. Scand J Gastroenterol 1985, 20 (Suppl. 107): 8-23.

10. Palmer, K.R., Berry, H., Wheeler, P.J. et al. Collagenous colitis - a relapsing and remitting disease. Gut 1986, 27: 578-580.

11. Kingham, J.G.C., Levison, D.A., Morson, B.C. \& Dawson, A.M. Collagenous colitis. Gut 1986, 27: 570-577.

12. Pascal, R.R., Kaye, G.I. \& Lane, N. Colonic pericryptal fibroblast sheath; replication, migration and cytodifferentiation of a mesenchymal cell systeme in adult tissue. I; autoradiographic studies of normal rabbit colon. Gastroenterology 1968, 54: 835-851. 